\title{
Cholesterol Modulates Rat Renal Brush Border Membrane Phosphate Transport
}

\author{
Moshe Levi, Becky M. Baird, and Paul V. Wilson \\ Department of Internal Medicine, Dallas Veterans Administration Medical Center, and University of Texas, \\ Southwestern Medical Center at Dallas, Dallas, Texas 75216
}

\begin{abstract}
In dietary phosphate $(\mathrm{Pi})$ deprivation and in aging there is an inverse correlation between renal proximal tubular brush border membrane (BBM) cholesterol (Chol) content, BBM fluidity, and BBM sodium gradient-dependent Pi transport activity (Na-Pi cotransport). The purpose of this study was to determine whether in vitro enrichment of renal BBM with Chol has a direct modulating effect on Na-Pi cotransport. 12 and 24 mol \% increases in Chol content caused dose-dependent decreases in $\mathrm{Na}-\mathrm{Pi}$ cotransport activity, 2,000 in control, vs. 1,450 in Chol $(+12 \%)$, vs. $900 \mathrm{pmol} / 5 \mathrm{~s} / \mathrm{mg}$ BBM protein in Chol (+24\%), all $P<0.01$, which was paralleled by dose-dependent increases in the fluorescence anisotropy of diphenylhexatriene, $r_{D P H}$, i.e., decrease in BBM fluidity, 0.203 in control, vs. 0.210 in Chol $(+12 \%)$, vs. 0.219 in Chol $(+24 \%)$, all $P$ $<0.01$. We found that increasing ambient temperature, which increases BBM fluidity independent of changes in Chol content, increased $\mathrm{Na}-\mathrm{Pi}$ cotransport. When $\mathrm{Na}-\mathrm{Pi}$ cotransport was analyzed as a function of $B B M$ fluidity, $1 / r_{D P H}$, we found that at an equivalent BBM fluidity BBM Chol enrichment still resulted in a dose-dependent decrease in $\mathrm{Na}-\mathrm{Pi}$ cotransport. Finally, in BBM isolated from rats fed a low Pi diet in vitro enrichment with Chol completely reversed the adaptive increases in $\mathrm{Na}-\mathrm{Pi}$ cotransport and fluidity. Our study therefore, indicates that $\mathrm{Chol}$ is a direct modulator of renal BBM Na-Pi cotransport activity, and that in vivo alterations in BBM Chol content most likely plays an important role in the regulation of renal tubular Pi transport. (J. Clin. Invest. 1990. 85:231-237.) acid binding $\bullet$ brush border membrane $\bullet$ cholesterol $\bullet$ fluidity $\bullet$ $\mathrm{Na}-\mathrm{Pi}$ cotransport $\bullet$ phosphonoformic
\end{abstract}

\section{Introduction}

Cholesterol is a major constituent of the plasma membrane (1). Changes in the cholesterol content of biological membranes alter the fluidity of the lipid bilayer (2), and concurrently influence a variety of plasma membrane functions including enzyme activity and ion transport processes (3-6). Specifically, the role of in vivo and in vitro alterations in cell membrane cholesterol content and/or fluidity in the modulation of Na,K-ATPase activity has been well studied (7-9). Recent studies indicate that alterations in renal proximal tubular brush border membrane (BBM) ${ }^{1}$ cholesterol content may also

Address reprint requests to Dr. Levi, Dallas VA Medical Center, Nephrology Service (111G1), 4500 South Lancaster Road, Dallas, TX 75216.

Received for publication 6 January 1989 and in revised form 24 August 1989

1. Abbreviations used in this paper: BBM, brush border membrane; BBMV, BBM vesicles; Pi, inorganic phosphate; PFA, phosphonoformic acid.

The Journal of Clinical Investigation, Inc.

Volume 85, January 1990, 231-237 play an important role in the regulation of sodium gradientdependent phosphate transport (Na-Pi cotransport). The agerelated decrease in the $V_{\max }$ of renal BBM Na-Pi cotransport (10) is associated with an age-related increase in BBM cholesterol content (11). Furthermore, the age-related impairment in the renal tubular adaptation to a low Pi diet is associated with the inability to lower BBM cholesterol content (11). In contrast, in young adult rats fed a low Pi diet the adaptive increase in the $V_{\max }$ of BBM Na-Pi cotransport (12) is associated with a selective decrease in BBM cholesterol content (13).

Although these in vivo studies suggest an important physiologic role for cholesterol in the regulation of Na-Pi cotransport, they do not necessarily establish a definite cause and effect relationship between an increase in BBM cholesterol content and a decrease in $\mathrm{Na}-\mathrm{Pi}$ cotransport activity. In addition, since in each case the alteration in BBM cholesterol content is paralleled by a change in BBM fluidity, it is also not clear whether cholesterol modulates $\mathrm{Na}$-Pi cotransport activity directly or through its effects on BBM fluidity. The purposes of the present study were, therefore, to determine $(a)$ whether in vitro alteration in BBM cholesterol content has a direct modulating effect on Na-Pi cotransport activity, $(b)$ whether cholesterol and/or fluidity per se or both modulate BBM Na-Pi cotransport activity, and $(c)$ whether in rats fed a low Pi diet, in vitro cholesterol enrichment reverses the in vivo adaptive increase in BBM Na-Pi cotransport activity.

\section{Methods}

Animals. All studies were performed in male Sprague-Dawley rats (Harlan Industries, Indianapolis, IN). Before the studies, the rats were equilibrated on a control diet $(0.6 \% \mathrm{Pi}, 0.6 \% \mathrm{Ca}$, wt/wt; Teklad, Madison, WI) for a 2-wk period. In studies where the effect of in vitro cholesterol enrichment on the in vivo adaptation to a low Pi diet was determined, the rats were fed a low Pi diet $(0.1 \% \mathrm{Pi}, 0.6 \% \mathrm{Ca})$ for a 1-wk period.

$B B M$ isolation. BBM from the renal superficial cortex were isolated by a differential centrifugation and magnesium precipitation method exactly as previously described from our laboratory $(11,14)$. Protein was determined by the method of Lowry et al. (15) using crystalline BSA as standard. Enzyme activity measurements including alkaline phosphatase, maltase, leucine aminopeptidase, and $\gamma$-glutamyl transferase (BBM-specific), and Na,K-ATPase (basolateral membrane-specific) were measured by kinetic spectrophotometric techniques on homogenate and BBM fractions to determine the specific activity and enrichment factor (specific activity in BBM fraction/specific activity in homogenate) of each BBM preparation $(11,14)$. Typically the BBM were enriched at least 12.0-fold for alkaline phosphatase, maltase, leucine aminopeptidase, and $\gamma$-glutamyl transferase and $<1.3$-fold for Na,K-ATPase.

Modulation of BBM cholesterol content. In vitro increase in BBM cholesterol content was achieved by incorporation of cholesteryl hemisuccinate (CHS) (Steraloids Inc., Pawling, NY), a hydrophilic cholesterol ester, by a slight modification of methods previously described (16). Briefly, freshly isolated BBM were suspended in 3.5\% polyvinylpyrrolidone, $1.0 \%$ BSA, $0.5 \%$ glucose in PBS, pH 7.50. Ethanol or CHS freshly dissolved in ethanol were then added, and the $\mathrm{BBM}$ were incubated in a shaking water bath at $37^{\circ} \mathrm{C}$ for $60 \mathrm{~min}$. In 
additional experiments to determine for nonspecific sticking rather than specific incorporation of the lipid to the BBM, cholesterol freshly dissolved in ethanol was also added. BBM were then washed three times in an ice-cold intravesicular buffer consisting of $300 \mathrm{mM}$ man-

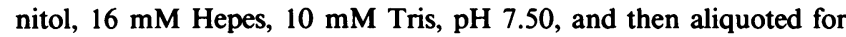
simultaneous measurements of $(a)$ lipid composition, (b) fluidity, (c) $\mathrm{Na}-\mathrm{Pi}$ cotransport activity, $(d) \mathrm{Na}$ gradient-dependent phosphonoformic acid (Na-PFA) binding, and (e) enzyme activity.

BBM lipid composition measurements. Total lipids were extracted by the method Bligh and Dyer (17). Coprostanol (Supelco, Inc., Bellefonte, PA) was added as an internal standard for cholesterol determination. To determine the BBM total cholesterol content, i.e., free cholesterol plus cholesteryl ester, an aliquot of the BBM sample was subjected to alkaline hydrolysis in a reaction mixture consisting of $0.3 \mathrm{ml}$ of $33 \% \mathrm{KOH}$ and $3.0 \mathrm{ml}$ of $88 \%$ ethanol, at $70^{\circ} \mathrm{C}$ for $30 \mathrm{~min}(18)$. Cholesterol content was determined by gas chromatography as previously described from our laboratory $(11,14)$. BBM cholesterol content was also determined by measuring free cholesterol and cholestryl ester content separately. Free and esterified cholesterol were separated by a one-dimensional TLC (silica gel; Eastman Kodak, Rochester, NY) with a solvent system which consisted of heptane/diethylether/ glacial acetic acid (85:15:2, vol/vol) (19). The amount of cholesterol in the cholesterol and cholesteryl ester spots were then measured as above. Individual phospholipid polar head group species were separated by a two-dimensional TLC (20) (silica gel 60; E. Merck, Darmstadt, West Germany) as previously described from our laboratory (11, 14). Phospholipid content in the total and individual phospholipid extract was determined by measuring the phosphorus content by the method of Ames and Dubin (21).

$B B M$ fluidity measurements. The effect of cholesterol enrichment on BBM fluidity was determined by measuring the steady-state and time-resolved fluorescence polarization of 1,6-diphenyl-1,3,5-hexatriene (DPH; Molecular Probes, Eugene, OR). BBM samples corresponding to $0.3 \mathrm{mg}$ BBM protein were diluted with a phosphate- and Hepes-buffered saline solution, $\mathrm{pH} 7.4$, to a concentration of $0.48 \mathrm{mg}$ protein $/ \mathrm{ml}$. $1 \mu \mathrm{l}$ of the lipid fluorophore was then added from a $1-\mathrm{mM}$ stock solution resulting in a probe/lipid ratio of $1: 300$. The steady-state fluorescence anisotropy $\left(r_{\mathrm{DPH}}\right)$ was measured using a photon counting spectrofluorometer (SLM 8000, SLM Instruments, Inc., Urbana, IL) as previously described from our laboratory (11). The parameter $r$ provides an index that is inversely related to membrane fluidity (22). The fluorescence lifetime of DPH was determined by time-resolved fluorescence measurements using a multifrequency phase and modulation fluorometer based on the Gratton design (23), as previously described (24). The measured phase and modulation values were analyzed assuming either that the decay was made up of a discrete sum of exponential components, or that the decay was made up of one or more continuous distributions of lifetime components (25-27).

$B B M$ phosphate transport measurements. Transport measurements were performed in freshly isolated BBM vesicles (BBMV) by a radiotracer uptake and rapid millipore filtration technique $(10,12)$. To determine $\mathrm{Na}^{+}$gradient-dependent ${ }^{32} \mathrm{Pi}$ uptake, $10 \mu \mathrm{l}$ of $\mathrm{BBMV}$, which were preloaded with a $300-\mathrm{mM}$ mannitol, Hepes-Tris, $\mathrm{pH} 7.50$ intravesicular buffer were vortex mixed with $40 \mu \mathrm{l}$ of an extravesicular buffer consisting of $100 \mathrm{mM}$ mannitol, $100 \mathrm{mM} \mathrm{NaCl}$, Hepes-Tris, pH 7.50, and $100 \mu \mathrm{M} \mathrm{K}_{2} \mathrm{H}^{32} \mathrm{PO}_{4}$. To determine non- $\mathrm{Na}^{+}$gradient-dependent or diffusive Pi uptake, $100 \mathrm{mM} \mathrm{NaCl}$ was replaced with $100 \mathrm{mM}$ $\mathrm{KCl}$. Uptake was terminated by the stop solution, which consisted of $135 \mathrm{mM} \mathrm{NaCl}, 10 \mathrm{mM} \mathrm{Na}_{2}$ arsenate, Hepes-Tris, pH 7.50.

BBM phosphonoformic acid binding measurements. To determine the effects of cholesterol enrichment on the number and affinity of the $\mathrm{Na}-\mathrm{Pi}$ cotransport units, $\mathrm{Na}^{+}$gradient-dependent equilibrium binding of phosphonoformic acid (Na-PFA) was determined by a slight modifcation of methods previously described (28). Briefly, BBMV were preloaded with the 300-mM mannitol, Hepes-Tris, pH 7.50 buffer. Binding was initiated in the presence of a $100 \mathrm{mM} \mathrm{NaCl}, 100 \mathrm{mM}$ mannitol, $0.2-12.8 \mathrm{mM}\left[{ }^{14} \mathrm{C}\right] \mathrm{PFA}$, Hepes-Tris, pH 7.50 solution. Maximal binding occurred at $30 \mathrm{~min}$, and binding was terminated with addition of an ice-cold stop solution, which consisted of $150 \mathrm{mM} \mathrm{NaCl}$, HepesTris, pH 7.50, followed by rapid Millipore filtration. The $V_{\max }$ and $K_{\mathrm{m}}$ of the equilibrium binding were determined according to the EadieScatchard transformation of the binding data (29).

Statistical analyses. All the data were expressed as mean \pm SE. A one-way analysis of variance with Student-Newman-Keuls multiple range test and/or a two-tailed unpaired Student's $t$ test were used to compare results between the control and the cholesterol-enriched BBM (30). Significance was accepted at $P<0.05$.

\section{Results}

The effect of in vitro cholesterol enrichment on brush border membrane lipid composition. As per the design of the study to achieve increases in BBM cholesterol content, which would simulate the $18 \%$ increase in cholesterol content in BBM derived from the aged rat (9), the in vitro incubations with CHS resulted in 12 and $24 \mathrm{~mol} \%$ increases in BBM cholesterol content and BBM cholesterol to phospholipid molar ratio (Table I). The in vitro incubations with CHS resulted in a selective alteration in BBM cholesterol composition, as there were no significant changes in BBM total (Table I) or individual phospholipid composition (results not shown).

The measured increase in BBM cholesterol content indicates incorporation of CHS into the BBM lipid bilayer rather than nonspecific sticking, as when BBM were similarly incubated with equimolar amounts of cholesterol rather than CHS, the resultant percent increases in BBM cholesterol content were $1.1 \%$ and $2.4 \%$ respectively, which indicates that nonspecific sticking accounts for $<10 \%$ of the measured increase in BBM cholesterol content.

The effect of in vitro cholesterol enrichment on brush border membrane transport activity. The in vitro increases in BBM cholesterol content of $12 \%$ and $24 \%$ caused dose-dependent

Table I. Effect of In Vitro Cholesterol on Brush Border Membrane Lipid Composition

\begin{tabular}{lccc}
\hline & Cholesterol & Total phospholipid & Cholesterol \\
& $n$ Tol/mg BBM protein & nmol/mg BBM protein & \\
& $408.8 \pm 12.2$ & $501.6 \pm 12.1$ & $0.815 \pm 0.016$ \\
Cholesterol $(+0 \%)$ & $459.5 \pm 17.3^{*}$ & $500.1 \pm 11.9$ & $0.919 \pm 0.019^{*}$ \\
Cholesterol $(+12 \%)$ & $510.6 \pm 18.4^{\ddagger 5}$ & $498.9 \pm 13.4$ & $1.023 \pm 0.020^{\ddagger 8}$
\end{tabular}

Results are expressed as mean $\pm \mathrm{SE}, n=6$ in each group. ${ }^{*} P<0.05$, cholesterol $(+12 \%)$ vs. cholesterol $(+0 \%),{ }^{\ddagger} P<0.05$, cholesterol $(+24 \%)$ vs. cholesterol $(+12 \%),{ }^{8} P<0.01$, cholesterol $(24 \%)$ vs. cholesterol $(+0 \%)$. 
decreases in BBMV Na-Pi cotransport activity (Fig. 1). The control as well as the cholesterol-enriched BBMV exhibited the "overshoot" phenomenon, which indicates time- and $\mathrm{Na}^{+}$ gradient-dependent concentrative uptake of $\mathrm{Pi}$. The initial rate (5 s) of $\mathrm{K}^{+}$gradient-dependent or diffusive uptake of Pi was $<5 \%$ of the Na-Pi cotransport activity in the control and cholesterol-enriched BBMV. The equilibrium $(2 \mathrm{~h})$ uptake of Pi was the same in all the three groups (Fig. 1), which indicates that intravesicular volume was not altered by the in vitro cholesterol enrichment. Further uptake experiments performed at $2,4,6,8$, and $10 \mathrm{~s}$ indicated that $\mathrm{Na}$-Pi cotransport was linear at least up to $10 \mathrm{~s}$. Extrapolation of uptake by linear regression to $0 \mathrm{~s}$ also indicated that binding of $\mathrm{Pi}$ is negligible in all the three groups and that differences in uptake represent true intravesicular transport of Pi (results not shown).

The differences in the initial rate $(5 \mathrm{~s})$ of Na-Pi cotransport activity between the control and the cholesterol-enriched BBMV were independent of alterations in transmembrane potential difference, as in voltage-clamped BBMV $\left(K_{\text {in }}=K_{\text {out }}\right.$ $=50 \mathrm{mM} \mathrm{KCl}$ plus valinomycin, $10 \mu \mathrm{g} / \mathrm{mg}$ BBM protein), $\mathrm{Na}-\mathrm{Pi}$ cotransport activity was $1,159 \pm 68$ in control vs. $793 \pm 74$ in Chol $(+12 \%), P<0.05$, vs. $527 \pm 74 \mathrm{pmol} / 5 \mathrm{~s} / \mathrm{mg}$ BBM protein in Chol $(+24 \%), P<0.05$.

The effect of cholesterol enrichment to decrease Na-Pi cotransport activity was shown not to be caused by a faster dissipation of the $\mathrm{Na}^{+}$gradient by three independent experiments. First, in the presence of $5 \mathrm{mM}$ amiloride in the uptake solution, Na-Pi cotransport activity was $1,383 \pm 59$ in control vs. $975 \pm 49$ in Chol (+12\%), $P<0.05$, vs. $628 \pm 51 \mathrm{pmol} / 5 \mathrm{~s} / \mathrm{mg}$ BBM protein in Chol ( $+24 \%), P<0.05$. Second, in the presence of $\mathrm{Na}^{+}$, but in the absence of a $\mathrm{Na}^{+}$gradient, i.e., $\mathrm{Na}_{\text {in }}$ $=\mathrm{Na}_{\text {out }}=100 \mathrm{mM} \mathrm{NaCl}$ plus monensin, $5 \mu \mathrm{g} / \mathrm{mg} \mathrm{BBM}$ protein, Na-Pi cotransport activity was $148 \pm 0.07$ in control vs. $108 \pm 6$ in Chol $(+12 \%), P<0.05$, vs. $73 \pm 0.06 \mathrm{pmol} / 5 \mathrm{~s} / \mathrm{mg}$ BBM protein in Chol $(+24 \%), P<0.05$. Finally, the effect of cholesterol on $\mathrm{BBM} \mathrm{Na}{ }^{+}$transport was determined directly by measuring $\mathrm{Na}-\mathrm{H}$ exchange activity, and cholesterol enrichment was found not to significantly alter $\mathrm{Na}-\mathrm{H}$ exchange activity (Table II).

While the in vitro increase in BBM cholesterol content caused a dose-dependent decrease in Na-Pi cotransport activity, it did not significantly alter BBMV Na-glucose or Na-proline cotransport activities (Table II). In addition, cholesterol enrichment also did not significantly change the specific activities of BBM-bound alkaline phosphatase, maltase, leucine aminopeptidase, or $\gamma$-glutamyl transferase (Table III). Thus, the levels of BBM cholesterol enrichment achieved in this study had selective and specific modulating effects on $\mathrm{Na}-\mathrm{Pi}$ cotransport activity.

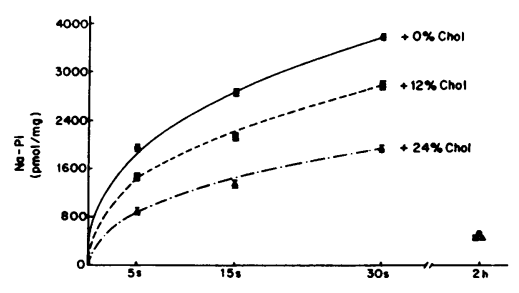

Figure 1. Dose-dependent effect of cholesterol enrichment on the time course of $\mathrm{Na}^{+}$gradient-dependent ${ }^{32} \mathrm{Pi}$ uptake (pmol/mg BBM protein), measured in the presence of intravesicular: $\mathbf{3 0 0} \mathrm{mM}$ man-

nitol, Hepes-Tris, pH 7.50, and extravesicular: $100 \mathrm{mM} \mathrm{NaCl}, 100$ $\mathrm{mM}$ mannitol, Hepes-Tris, pH 7.50, $100 \mu \mathrm{M} \mathrm{K}_{2} \mathrm{H}^{32} \mathrm{PO}_{4}$. Results are expressed as mean \pm SE. $n=6$ in each group.
Table II. Effect of In Vitro Cholesterol on Brush Border Membrane Transport

\begin{tabular}{llcc}
\hline & \multicolumn{1}{c}{ Na-H } & Na-Glucose & Na-Proline \\
\hline & pmol/5 s/mg & pmol/5 s/mg & pmol/5 s/mg \\
Cholesterol (+0\%) & $1,466 \pm 106$ & $202 \pm 21$ & $198 \pm 12$ \\
Cholesterol (+12\%) & $1,378 \pm 96$ & $199 \pm 17$ & $218 \pm 17$ \\
Cholesterol (+24\%) & $1,335 \pm 112$ & $196 \pm 16$ & $184 \pm 19$
\end{tabular}

Results are expressed as mean $\pm \mathrm{SE}, n=4$ in each group. $\mathrm{pH}$ gradient-dependent $\mathrm{Na}$ uptake (Na-H exchange) was measured by vortex mixing $10 \mu \mathrm{l}$ of BBMV preloaded with a $150 \mathrm{mM} \mathrm{KCl}, 25 \mathrm{mM}$ MES, pH 5.50 intravesicular buffer with $40 \mu \mathrm{l}$ of uptake solution which consisted of $1 \mathrm{mM}^{22} \mathrm{NaCl}, 149 \mathrm{mM} \mathrm{KCl}, 25 \mathrm{mM}$ Hepes, $\mathrm{pH}$ $7.50, \pm 1 \mathrm{mM}$ amiloride. Uptake was terminated by the stop solution which consisted of $150 \mathrm{mM} \mathrm{KCl}, 25 \mathrm{mM}$ Hepes, $0.1 \mathrm{mM}$ Amiloride, pH 7.50. $\mathrm{Na}^{+}$gradient-dependent glucose and proline uptake were measured by vortex mixing $10 \mu \mathrm{l}$ of BBMV preloaded with $300 \mathrm{mM}$ mannitol, Hepes-Tris, pH 7.50 intravesicular buffer with $40 \mu \mathrm{l}$ of uptake solution, which consisted of $100 \mathrm{mM} \mathrm{NaCl}, 100 \mathrm{mM}$ mannitol, Hepes-Tris, pH 7.50, and $50 \mu \mathrm{M}\left[{ }^{3} \mathrm{H}\right]-\mathrm{D}$-glucose or $25 \mu \mathrm{M}\left[{ }^{3} \mathrm{H}\right]-\mathrm{L}-$ proline. Uptake was terminated by the stop solution, which consisted of $150 \mathrm{mM} \mathrm{NaCl}, 0.25 \mathrm{mM}$ phloridzin, Hepes-Tris, $\mathrm{pH} 7.50$.

The effect of in vitro cholesterol enrichment on brush border membrane Na-Pi cotransport kinetics. To determine whether cholesterol enrichment caused a decrease in Na-Pi cotransport activity by altering either the binding of $\mathrm{Na}^{+}$to the $\mathrm{Na}-\mathrm{Pi}$ cotransporter and/or the stoichiometry of Na-Pi cotransport, the initial rate of ${ }^{32} \mathrm{Pi}$ uptake was measured as a function of extravesicular $\mathrm{Na}^{+}$ranging from 0 to $150 \mathrm{mM} \mathrm{NaCl}$. A sigmoidal relationship between $\mathrm{Pi}$ uptake and extravesicular $\mathrm{Na}^{+}$ concentration was observed for both the control and cholesterol-enriched BBM (Fig. 2). Kinetic analysis of the Hill plot indicated a coefficient $(n)$ of 1.82 for control, 1.91 for $+12 \%$ Chol, and 1.86 for $+24 \%$ Chol BBM, which indicates the interaction of at least two $\mathrm{Na}^{+}$ions with the $\mathrm{Na}-\mathrm{Pi}$ cotransporter, and that cholesterol enrichment did not significantly alter the stoichiometry of the $\mathrm{Na}-\mathrm{Pi}$ cotransporter. In addition, when the data in Fig. 2 were analyzed according to the Wolf-Augustinsson-Hofstee linear transformation of the Michaelis-Menten equation (29), the best fit in linear regression analysis was obtained with the $\mathrm{Na}$ concentration expressed as [Na] ${ }^{2}$ (Fig. 2, inset). Kinetic analysis of the data yielded $K_{\mathrm{Na}}$ values of $35 \pm 2$ $\mathrm{mM}$ in control, $33 \pm 3 \mathrm{mM}$ in $+12 \% \mathrm{Chol}$, and $32 \pm 3 \mathrm{mM} \mathrm{Na}$ in $+24 \%$ Chol, which indicates that cholesterol enrichment did not alter the binding of $\mathrm{Na}^{+}$to the $\mathrm{Na}-\mathrm{Pi}$ cotransporter.

The effect of cholesterol enrichment on the kinetics of $\mathrm{Na}-\mathrm{Pi}$ cotransport was further analyzed by measuring the initial rate of ${ }^{32} \mathrm{Pi}$ uptake in the presence of $100 \mathrm{mM} \mathrm{NaCl}$ and as a function of extravesicular Pi ranging from 25 to $800 \mu \mathrm{M} P i$ (Fig. 3). Analysis of the data according to the Wolf-Augustinsson-Hofstee linear transformation of the Michaelis-Menten equation (29) indicated that cholesterol enrichment decreased the $V_{\max }$ of $\mathrm{Na}-\mathrm{Pi}$ cotransport, $4,380 \pm 100$ in control vs. $3,100 \pm 120$ in $+12 \%$ Chol, $P<0.01$, vs. $1,550 \pm 100 \mathrm{pmol} \mathrm{Pi} / 5$ $\mathrm{s} / \mathrm{mg}$ BBM protein in $+24 \%$ Chol, $P<0.01$, whereas cholesterol enrichment did not alter the $K_{\mathrm{Pi}}, 132 \pm 6$ in control vs. $131 \pm 4$ in $+12 \%$ Chol, $P=$ NS, vs. $128 \pm 6 \mu \mathrm{M}$ Pi in $+24 \%$ Chol, $P=$ NS (Fig. 3 inset). 
Table III. Effect of In Vitro Cholesterol on BBM Enzyme Activity

\begin{tabular}{lcccc}
\hline & Alkaline phosphatase & Maltase & Leucine aminopeptidase & \multicolumn{1}{c}{$\gamma$-Glutamyl transferase } \\
\hline & $\mu m o l / h$ per $m g$ & $\mu m o l / h$ per $m g$ & $\mu m o l / h$ per $m g$ & $\mu m o l / h$ per $m g$ \\
Cholesterol $(+0 \%)$ & $977 \pm 49$ & $228 \pm 14$ & $52 \pm 8$ & $1,951 \pm 74$ \\
Cholesterol $(+12 \%)$ & $981 \pm 61$ & $244 \pm 12$ & $56 \pm 9$ & $1,962 \pm 81$ \\
Cholesterol $(+24 \%)$ & $950 \pm 59$ & $252 \pm 18$ & $57 \pm 7$ & $1,873 \pm 89$
\end{tabular}

Results are expressed as mean $\pm \mathrm{SE}, n=4$ in each group.

The effect of in vitro cholesterol enrichment on brush border membrane $\mathrm{Na}-\mathrm{Pi}$ carrier number and/or accessibility. To determine whether cholesterol enrichment caused a decrease in the $V_{\max }$ of Na-Pi cotransport activity by causing a decrease in the number and/or accessibility of the $\mathrm{Na}$-Pi carrier units, $\mathrm{Na}^{+}$ gradient-dependent equilibrium binding of $\left[{ }^{14} \mathrm{C}\right] \mathrm{PFA}$ was measured. Eadie-Scatchard analysis of the equilibrium binding data indicated that cholesterol enrichment did not alter the $V_{\max }, 21.4 \pm 1.8$ in control vs. $20.6 \pm 2.7$ in $+12 \%$ Chol, $P=\mathrm{NS}$, vs. $23.3 \pm 2.2 \mathrm{nmol} \mathrm{PFA} / 30 \mathrm{~min} / \mathrm{mg}$ BBM protein in $+24 \%$ Chol, $P=\mathrm{NS}$, or the $\mathrm{K}_{\mathrm{PFA}}, 4.1 \pm 0.9$ in control vs. $3.7 \pm 1.2$ in $+12 \%$ Chol, $P=$ NS, vs. $4.3 \pm 0.8$ mM PFA in $+24 \%$ Chol, $P$ = NS.

The effect of in vitro cholesterol enrichment on brush border membrane fluidity. The dose-dependent effect of cholesterol enrichment to decrease the initial rate of $\mathrm{Na}-\mathrm{Pi}$ cotransport activity was paralleled by the dose-dependent effect of cholesterol enrichment to increase the steady-state fluorescence anisotropy of DPH, $r_{\mathrm{DPH}}$ (Table IV). Analysis of the time-re-

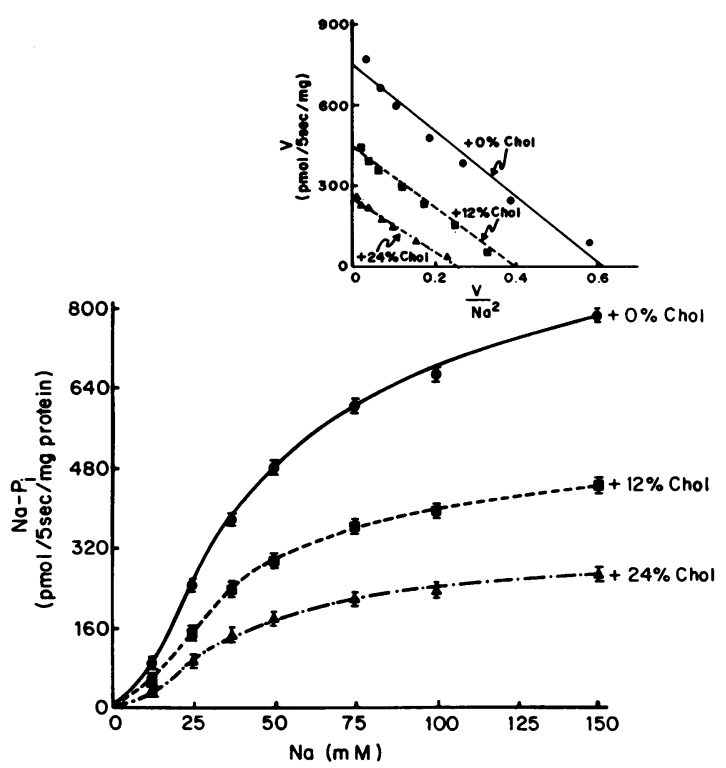

Figure 2. The effect of varying extravesicular $\mathrm{Na}^{+}$concentration $(\mathrm{mM})$ on the initial rate of ${ }^{32} \mathrm{Pi}$ uptake (pmol/5 $\mathrm{s} / \mathrm{mg}$ BBM protein), measured in the presence of intravesicular: $300 \mathrm{mM}$ mannitol, Hepes-Tris, pH 7.50, and extravesicular: 0 to $150 \mathrm{mM} \mathrm{NaCl}$, HepesTris, $\mathrm{pH} 7.50,100 \mu \mathrm{M} \mathrm{K}_{2} \mathrm{H}^{32} \mathrm{PO}_{4}$. $\mathrm{NaCl}$ was isomotically replaced with choline chloride. Results are expressed as mean \pm SE. $n=6$ in each group. The insert shows the Woolf-Augustinsson-Hofstee transformation of the data assuming a Hill coefficient of 2.0 for $\mathrm{Na}^{+}$. The coefficient of correlation in each group was 0.97-0.99. solved fluorescence measurements indicated that the phase and modulation lifetimes exhibited a small deviation $(<5 \%)$ from a single-exponential decay, as is typically found for DPH in membrane systems above the lipid phase transition (25-27). The average lifetimes, 9.9 in control, vs. 10.0 in $+12 \%$ cholesterol, vs. 10.1 ns in $+24 \%$ cholesterol, were not significantly different between the control and the cholesterol-enriched BBM. Hence, the increase in $r_{\mathrm{DPH}}$ as a function of cholesterol enrichment reflects a decrease in BBM fluidity, rather than a decrease in the lifetime of the fluorophore.

The effect of temperature on BBM fluidity and $\mathrm{Na}-\mathrm{Pi}$ cotransport activity. BBM fluidity and the initial rate of $\mathrm{Na}-\mathrm{Pi}$ cotransport activity were also measured as a function of ambient temperature. Increasing ambient temperature from $25^{\circ}$ to $37^{\circ} \mathrm{C}$ (which is above the lipid phase transition for rat renal $\mathrm{BBM}$ ) resulted in parallel increases in BBM fluidity (decrease in $r_{\mathrm{DPH}}$ ) and $\mathrm{Na}$-Pi cotransport activity (Table IV). Analysis of the data indicates that increasing BBM fluidity, i.e., increasing $1 / r_{\mathrm{DPH}}$, causes an increase in $\mathrm{Na}-\mathrm{Pi}$ cotransport activity. In

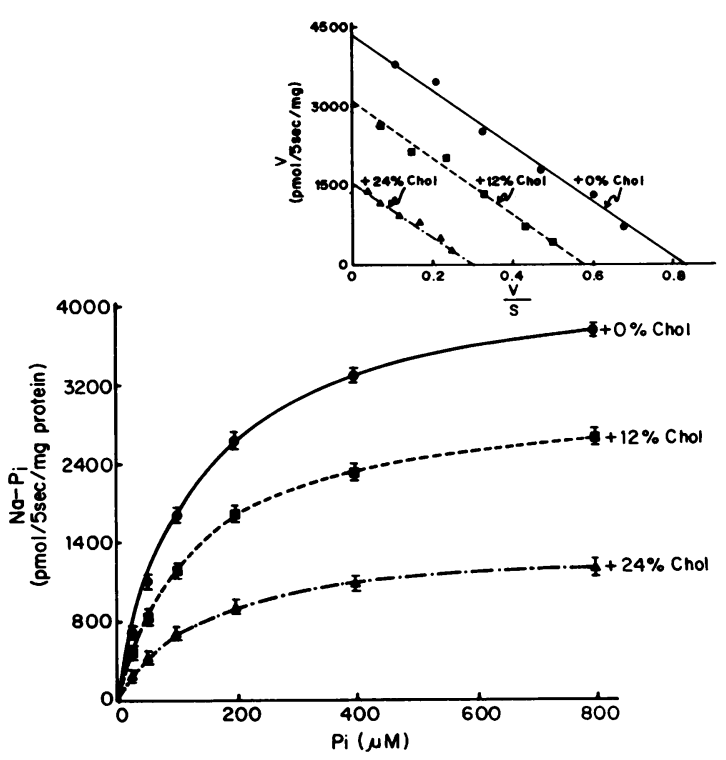

Figure 3. The effect of varying extravesicular Pi concentration $(\mu \mathrm{M})$ on the initial rate of $\mathrm{Na}^{+}$gradient-dependent ${ }^{32} \mathrm{Pi}$ uptake (pmol/5 $\mathrm{s} / \mathrm{mg}$ BBM protein), measured in the presence of intravesicular: 300 $\mathrm{mM}$ mannitol, Hepes-Tris, 7.50 , and extravesicular: $100 \mathrm{mM} \mathrm{NaCl}$, $100 \mathrm{mM}$ mannitol, Hepes-Tris, 7.50, 25-800 $\mu \mathrm{M} \mathrm{K}_{2} \mathrm{H}^{32} \mathrm{PO}_{4}$. Results are expressed as mean \pm SE. $n=6$ in each group. The insert shows the Woolf-Augustinsson-Hofstee transformation of the data. The coefficient of correlation in each group was 0.97-0.99. 
Table IV. Effect of In Vitro Cholesterol on Brush Border Membrane Fluidity and Na-Pi Cotransport Activity as a Function of Temperature

\begin{tabular}{lcc}
\hline & Anisotropy $\left(r_{\mathrm{DPH}}\right)$ & $\mathrm{Na}-\mathrm{Pi}$ \\
\hline & & pmol/5 s/mg BBM protein \\
$25^{\circ} \mathrm{C}$ & & \\
$\mathrm{Chol}(+0 \%)$ & $0.255 \pm 0.001$ & $1,483 \pm 69$ \\
$\mathrm{Chol}(+12 \%)$ & $0.260 \pm 0.001^{*}$ & $1,075 \pm 59^{*}$ \\
$\mathrm{Chol}(+24 \%)$ & $0.265 \pm 0.001^{\ddagger \S}$ & $728 \pm 61^{\ddagger \S}$ \\
$31^{\circ} \mathrm{C}$ & & \\
$\mathrm{Chol}(+0 \%)$ & $0.240 \pm 0.001$ & $1,665 \pm 64$ \\
$\mathrm{Chol}(+12 \%)$ & $0.245 \pm 0.001^{*}$ & $1,366 \pm 74^{*}$ \\
$\mathrm{Chol}(+24 \%)$ & $0.249 \pm 0.001^{\ddagger \S}$ & $1,023 \pm 81^{\ddagger \S}$ \\
$37^{\circ} \mathrm{C}$ & & \\
Chol $(+0 \%)$ & $0.220 \pm 0.001$ & $1,925 \pm 55$ \\
Chol $(+12 \%)$ & $0.225 \pm 0.001^{*}$ & $1,722 \pm 59^{*}$ \\
Chol $(+24 \%)$ & $0.230 \pm 0: 001^{\ddagger 5}$ & $1,381 \pm 71^{\ddagger \S}$ \\
\hline
\end{tabular}

Results are expressed as mean \pm SE, $n=4$ in each group. ${ }^{*} P<0.05$, cholesterol $(+12 \%)$ vs. cholesterol $(+0 \%),{ }^{\ddagger} P<0.05$, cholesterol $(+24 \%)$ vs. cholesterol $(+12 \%),{ }^{\S} P<0.01$, cholesterol $(+24 \%)$ vs. cholesterol $(+0 \%)$.

fact, when Na-Pi cotransport activity is plotted as a function of $1 / r_{\mathrm{DPH}}$, there is a highly linear relationship $(r>0.98$, results not shown). Further analysis of the data also indicates that for a given identical BBM fluidity, for example, $r_{\mathrm{DPH}} 0.242$ or $1 / r_{\mathrm{DPH}} 4.125$, BBM cholesterol enrichment still causes a decrease in Na-Pi cotransport activity, 1,630 164 in control, vs. $1,389 \pm 71$ in $+12 \%$ Chol, $P<0.01$, vs. $1,133 \pm 59 \mathrm{pmol} / 5 \mathrm{~s} / \mathrm{mg}$ BBM protein in $+24 \%$ Chol, $P<0.01$.

The effect of in vitro cholesterol enrichment on $\mathrm{BBM} \mathrm{Na-Pi}$ cotransport and fluidity in rats fed a low-phosphate diet. In agreement with the earlier studies (11-13), we found that in rats fed a low Pi diet, there were significant increases in BBM $\mathrm{Na}$-Pi cotransport activity and BBM fluidity (Fig. 4). Since in rats fed a low Pi diet, the increase in BBM fluidity is caused by a selective decrease in $\mathrm{BBM}$ cholesterol content $(11,13)$, we
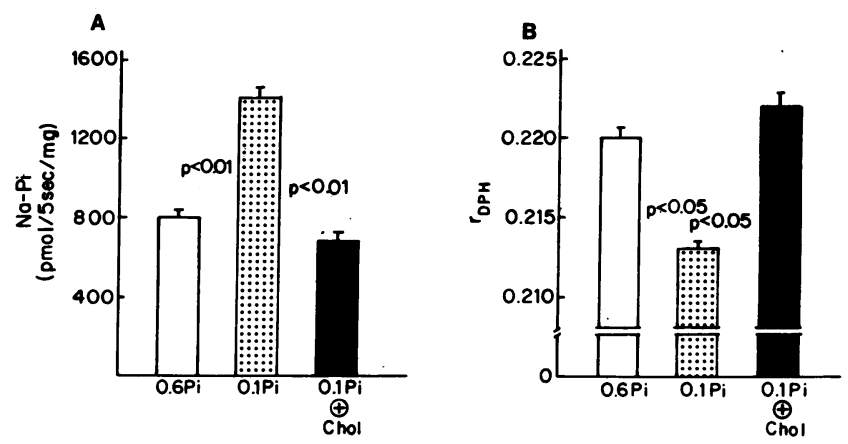

Figure 4. Effects of cholesterol enrichment on the adaptive increase in the initial rate of $\mathrm{Na}^{+}$gradient-dependent ${ }^{32} \mathrm{Pi}$ uptake $(\mathrm{Na}-\mathrm{Pi}$, pmol/5 s/mg BBM protein, $A$ ), and the adaptive decrease in the fluorescence anisotropy of diphenylhexatriene $\left(r_{\mathrm{DPH}}, B\right)$ in brush border membranes isolated from rats fed a low Pi diet. Results are expressed as mean \pm SE. $n=4$ in each group. wished to determine whether in vitro enrichment of these $B B M$ with cholesterol would then reverse the adaptive increases in BBM Na-Pi cotransport activity and BBM fluidity. Indeed, when BBM isolated from rats fed a low Pi diet were incubated with CHS to result in BBM cholesterol content, which was the same as the cholesterol content of BBM isolated from rats fed a control Pi diet, there was a complete reversal of BBM Na-Pi cotransport activity and fluidity to the same levels as measured in the BBM isolated from rats fed a control Pi diet (Fig. 4).

\section{Discussion}

In vivo and in vitro alterations in the $V_{\max }$ of enzyme and transport protein activity, including $\mathrm{Na}-\mathrm{Pi}$, have been proposed to be mediated by several mechanisms including alterations in de novo protein synthesis and/or degradation, protein exocytosis and/or endocytosis, protein phosphorylation and/or dephosphorylation, and protein ribosylation and/or deribosylation. Recent studies suggest that alterations in membrane lipid composition and/or lipid fluidity may also play an important role in the regulation of enzyme and transport protein activity (3-9), including renal BBM Na-Pi cotransport activity $(11,13,31,32)$. In our previous study we found that the age-related decrease in the $V_{\max }$ of $\mathrm{Na}-\mathrm{Pi}$ cotransport activity was correlated with an increase in BBM cholesterol content and a decrease in BBM fluidity, and that the increase in BBM cholesterol content could play a further role in the age-related impairment in the renal tubular adaptation to a low Pi diet (11). In this study we show that in BBM isolated from young adult rat in vitro enrichment with cholesterol, to an identical level observed in the aged rat, results in similar reductions in BBM fluidity and in the $V_{\max }$ of $\mathrm{Na}-\mathrm{Pi}$ cotransport activity. In addition, in BBM isolated from young adult rats adapted to a low $\mathrm{Pi}$ diet, in vitro enrichment with cholesterol completely reverses the adaptive increases in BBM fluidity and the $V_{\max }$ of $\mathrm{Na}-\mathrm{Pi}$ cotransport activity. Altogether, these results strongly indicate a physiologic role for BBM cholesterol content and/or fluidity in the regulation of renal tubular Na-Pi cotransport activity.

We found that the decrease in Na-Pi cotransport activity as a function of cholesterol enrichment is paralleled by a similar decrease in BBM fluidity. In the present study we did not measure the effects of cholesterol enrichment on the static and dynamic components of membrane fluidity separately, however, previous studies in artificial liposomes and biological membranes have indicated that in lipid bilayers with a low cholesterol to phospholipid $(<0.33)$ and/or saturated to unsaturated fatty acid molar ratio, cholesterol enrichment has a more pronounced effect on the static component of fluidity, whereas in lipid bilayers with a high cholesterol to phospholipid ( $>0.40)$ and/or saturated to unsaturated fatty acid molar ratio, such as the renal BBM, cholesterol enrichment has similar effects on both the static as well as the dynamic components of membrane fluidity (33-35).

As cholesterol enrichment has concurrent and similar effects on both Na-Pi cotransport activity and BBM fluidity, an interesting question is whether BBM cholesterol content and/ or fluidity per se or both modulate $\mathrm{Na}-\mathrm{Pi}$ cotransport activity. In trying to answer this question we took advantage of the fact that independent of changes in BBM cholesterol content, both 
BBM fluidity and $\mathrm{Na}-\mathrm{Pi}$ cotransport activity increase as a function of increasing ambient temperature. This allowed us to express $\mathrm{Na}-\mathrm{Pi}$ cotransport activity as a function of fluidity for each of the three BBM cholesterol groups. In this fashion, for a given level of identical BBM fluidity, we found that there was still a significant decrease in $\mathrm{Na}-\mathrm{Pi}$ cotransport activity as a function of increasing BBM cholesterol content. Thus, our analysis indicates that BBM fluidity and BBM cholesterol content each modulate $\mathrm{Na}-\mathrm{Pi}$ cotransport activity. Since the fluorescence anisotropy of DPH may largely reflect changes in bulk membrane fluidity, it is still possible that cholesterol may exert its effects through alterations in the fluidity of the lipid annulus (microdomain) surrounding the Na-Pi cotransporter (36). Alternatively, it is also likely that cholesterol may modulate the $\mathrm{Na}-\mathrm{Pi}$ cotransporter by directly binding to it, or through some other chemical modification of the transporter (6).

The $\left[{ }^{14} \mathrm{C}\right] \mathrm{PFA}$ binding and the Na-Pi uptake kinetic studies provide important insights into the mechanisms by which cholesterol enrichment modulates $\mathrm{Na}-\mathrm{Pi}$ cotransport activity. The $\left[{ }^{14} \mathrm{C}\right] \mathrm{PFA}$ binding studies indicate that cholesterol enrichment does not alter the number or the accessibility of the $\mathrm{Na}-\mathrm{Pi}$ cotransport units. Na-Pi uptake studies as a function of extravesicular [ $\mathrm{Na}]$ indicates that cholesterol enrichment does not alter the stoichiometry of $\mathrm{Na}$-Pi cotransport. In addition, Na-Pi uptake kinetic studies as a function of extravesicular [Na] and [Pi] also indicates that cholesterol enrichment does not alter the $K_{\mathrm{Na}}$ or $K_{\mathrm{Pi}}$. Thus cholesterol enrichment does not alter the binding of $\mathrm{Na}$ or $\mathrm{Pi}$ to the $\mathrm{Na}-\mathrm{Pi}$ carrier units. We propose that cholesterol enrichment most likely decreases the $V_{\max }$ of Na-Pi cotransport activity by decreasing the rate of movement of the carrier, i.e., turnover, once loaded with $\mathrm{Na}$ and $\mathrm{Pi}$.

In the present study we found that cholesterol enrichment selectively modulated $\mathrm{Na}-\mathrm{Pi}$ cotransport, as cholesterol enrichment had no significant effects on $\mathrm{Na}-\mathrm{H}$ exchange, or Na-glucose and Na-proline cotransport activities. The lack of the effect of increase in BBM cholesterol content and/or decrease in BBM fluidity on $\mathrm{Na}-\mathrm{H}$ exchange activity is in agreement with a previous study that showed that in isolated renal BBM $n$-alkanols, which caused a dose-dependent increase in BBM fluidity, did not influence $\mathrm{Na}-\mathrm{H}$ exchange activity (37). Similarly, the results of our study are in agreement with the findings in dietary Pi deprivation and in the aged rat where the alterations in BBM cholesterol content and/or fluidity were found to have selective effects on Na-Pi cotransport, and not to influence Na-glucose or Na-proline cotransport activities $(10,12)$. The effect of in vitro cholesterol enrichment on renal BBM $\mathrm{Na}$-glucose cotransport activity has not been previously determined. On the other hand, in LM cells (mouse fibroblasts) cholesterol depletion has been shown to reduce the $V_{\max }$ of 3-O-methyl-glucose transport with no change in $K_{\mathrm{m}}$, and subsequent cholesterol repletion in the same cells was shown to result in restoration of the $V_{\max }$ of glucose transport to baseline values $(38,39)$. In this respect the renal $B B M$ may differ from the LM cells. In renal BBM the effect of in vitro alterations in BBM fluidity on Na-glucose cotransport has been studied but the results are conflicting. Increasing ambient temperature, which increases BBM fluidity, has been shown to increase renal BBM Na-glucose cotransport, whereas incubation of BBM with either oleic acid (40), or benzyl alcohol $(41,42)$, which also increase BBM fluidity, has been shown to decrease renal BBM Na-glucose cotransport activity. Oleic acid, how- ever, causes a faster dissipation of the $\mathrm{Na}^{+}$gradient (40), and benzyl alcohol causes a decrease in the number of Na-dependent phloridzin binding sites (42). Therefore, the effects on $\mathrm{Na}^{+}$gradient-dissipation and the number or accessibility of glucose carrier units, rather than the effects on BBM fluidity per se, may mediate the inhibitory effects of oleic acid and benzyl alcohol on Na-glucose cotransport activity.

In this study we also found that cholesterol enrichment had no significant effects on BBM-bound alkaline phosphatase, maltase, leucine aminopeptidase, or $\gamma$-glutamyltransferase specific activities. The lack of effect of cholesterol enrichment on alkaline phosphatase activity, was actually surprising since in dietary $\mathrm{Pi}$ deprivation and in the aged rat the changes in BBM cholesterol content and fluidity were associated with specific alterations in the $V_{\max }$ of alkaline phosphatase activity $(11,14)$. In addition, in a recent study in small intestinal BBM in vitro alteration of BBM cholesterol content was shown to specifically modulate the $V_{\max }$ of alkaline phosphatase activity (43). We cannot readily explain these apparent differences, however, the renal and small intestinal alkaline phosphatase molecules are known to differ in catalytic and antigenic properties (44), and therefore could differ in their response to increases in BBM cholesterol content. In addition, in dietary $\mathrm{Pi}$ deprivation and in aging $(11,14)$, it is possible that the alterations in the $V_{\max }$ of alkaline phosphatase were mediated by other factors in addition to changes in BBM cholesterol content, such as alterations in the number of enzyme units. It is of interest that in small intestinal and renal BBM benzyl alcohol which increases BBM fluidity has been shown not to affect alkaline phosphatase activity $(41,43)$. Further studies are needed to determine if selective in vitro changes in BBM cholesterol content and/or fluidity regulate renal alkaline phosphatase activity.

In summary, the results of our study indicate that BBM cholesterol content is a direct modulator of $\mathrm{Na}-\mathrm{Pi}$ cotransport activity, and that in vivo alterations in BBM cholesterol content most likely play an important physiologic role in the regulation of renal tubular Pi transport in pathophysiologic conditions, including in aging and in dietary Pi deprivation.

\section{Acknowledgments}

The authors would like to thank Dr. David M. Jameson for his help and guidance with the fluorescence polarization and lifetime measurements, Dr. Biff Palmer for his help with the $\mathrm{Na}-\mathrm{H}$ exchange measurements, Ms. Regina Citizen and Ginny Mitchell for expert secretarial assistance, and the Medical Media Department at the Dallas VAMC for the illustrations.

This work was supported by grants from the Veterans Administration Research Service, the American Heart Association (Texas Affiliate), and the National Dairy Promotion and Research Board- administered by the National Dairy Council.

\section{References}

1. Cullis, P. R., and M. J. Hope. 1985. Physical properties and functional roles of lipids in membranes. In Biochemistry of Lipids and Membranes. D. E. Vance and J. E. Vance, editors. The Benjamin/ Cummings Publishing Co., Inc., Menlo Park, CA. 25-72.

2. van Blitterswijk, W. J., B. W. van der Meer, and H. Hilkmann. 1987. Quantitiative contributions of cholesterol and the individual classes of phospholipids and their degree of fatty acyl (un)saturation to membrane fluidity measured by fluorescence polarization. Biochemistry. 26:1746-1756.

3. Spector, A. A., and M. A. Yorek. 1985. Membrane lipid composition and cellular function. J. Lipid Res. 26:1015-1035. 
4. Deuticke, B., and C. W. M. Haest. 1987. Lipid modulation of transport proteins in vertebrate cell membranes. Annu. Rev. Physiol. 49:221-235.

5. Illsley, N. P., H. Y. Lin, and A. S. Verkman. 1987. Lipid domain structure correlated with membrane protein function in placental microvillus vesicles. Biochemistry. 26:446-454.

6. Yeagle, P. L. 1989. Lipid regulation of cell membrane structure and function. FASEB (Fed. Am. Soc. Exp. Biol.) J. 3:1833-1842.

7. Sinensky, M., F. Pinkerton, E. Sutherland, and F. R. Simon. 1979. Rate limitation of $\left(\mathrm{Na}^{+}+\mathrm{K}^{+}\right)$-stimulated adenosinetriphosphatase by membrane acyl chain ordering. Proc. Natl. Acad. Sci. USA. 76:4893-4897.

8. Keeffe, E. B., B. F. Scharschmidt, N. M. Blankenship, and R. K. Ockner. 1979. Studies of relationship among bile flow, liver plasma membrane $\mathrm{Na}, \mathrm{K}-\mathrm{ATPase}$, and membrane microviscosity in the rat. $J$. Clin. Invest. 64:1590-1598.

9. Yeagle, P. L., J. Young, and D. Rice. 1988. Effects of cholestero on $\left(\mathrm{Na}^{+}, \mathrm{K}^{+}\right)$-ATPase ATP hydrolyzing activity in bovine kidney. Biochemistry. 27:6449-6452.

10. Kiebzak, G. M., and B. Sacktor. 1986. Effect of age on renal conservation of phosphate in the rat. Am. J. Physiol. 251(Renal Fluid and Electrolyte Physiol. 20):F399-F407.

11. Levi, M., D. M. Jameson, and B. W. van der Meer. 1989. Role of BBM lipid composition and fluidity in impaired renal Pi transport in aged rat. Am. J. Physiol. 256(Renal Fluid and Electrolyte Physiol. 25):F85-F94.

12. Cheng, L., C. T. Liang, and B. Sacktor. 1983. Phosphate uptake by renal membrane vesicles of rabbits adapted to high and low phosphorus diets. Am. J. Physiol. 245(Renal Fluid and Electrolyte Physiol. 14):F175-F180.

13. Molitoris, B. A., A. C. Alfrey, R. A. Harris, and F. R. Simon. 1985. Renal apical membrane cholesterol and fluidity in regulation of phosphate transport. Am. J. Physiol. 243(Renal Fluid and Electrolyte Physiol. 18):F12-F19.

14. Levi, M., B. A. Molitoris, T. J. Burke, R. W. Schrier, and F. R. Simon. 1987. Effects of vitamin D-induced chronic hypercalcemia on rat renal cortical plasma membranes and mitochondria. Am. J. Physiol. 252(Renal Fluid and Electrolyte Physiol. 21):F267-F275.

15. Lowry, O. H., N. J. Rosebrough, A. L. Farr, and R. J. Randall. 1951. Protein measurements with the Folin phenol reagent. J. Biol. Chem. 193:265-275.

16. Yuli, I., W. Wilbrandt, and M. Shinitzky. 1981. Glucose transport through cell membranes of modified lipid fluidity. Biochemistry. 20:4250-4256.

17. Bligh, E. G., and W. J. Dyer. 1959. A rapid method of total lipid extraction and purification. Can. J. Biochem. Physiol. 37:911-917.

18. Spinedi, A., S. Rufini, P. Luly, and R. N. Farias. 1988. The temperature-dependence of human erythrocyte acetylcholinesterase activity is not affected by membrane cholesterol enrichment. Biochem. J. 255:547-551.

19. Slotte, J. P., and E. L. Bierman. 1988. Depletion of plasmamembrane sphingomyelin rapidly alters the distribution of cholesterol between plasma membranes and intracellular cholesterol pools in cultured fibroblasts. Biochem. J. 250:653-658.

20. Esko, J. D., and C. R. H. Raetz. 1980. Mutants of Chinese hamster ovary cells with altered membrane hospolipid composition. $J$. Biol. Chem. 255:4474-4480.

21. Ames, B. N., and D. T. Dubin. 1960 . The role of polyamines in the neutralization of bacteriophage of deoxyribonucleic acid. J. Biol. Chem. 235:769-775.

22. Jameson, D. M. 1984. Fluorescence: principles, methodologies, and applications. In Fluorescence Hapten: an Immunological Probe. E. W. Voss, Jr., editor. CRC Press, Boca Raton, FL. 23-48.

23. Gratton, E., and M. Limkeman. 1983. A continuously variable frequency cross-correlation phase fluorometer with picosecond resolution. Biophys. J. 44:315-324.

24. Jameson, D. M., E. Gratton, and J. F. Eccleston. 1987. Intrinsic fluorescence of elongation factor Tu in its complexes with GDP and elongation factor Ts. Biochemistry. 26:3894-3901.

25. Barrow, D. A., and B. R. Lentz. 1985. Membrane structural domains. Resolution limits using diphenylhexatriene fluorescence decay. Biophys. J. 48:221-234.

26. Fiorini, R., M. Valentino, S. Wang, M. Glaser, and E. Gratton. 1987. Fluorescence lifetime distributions of 1,6-diphenyl-1,3,5-hexatriene in phospholipid vesicles. Biochemistry. 26:3864-3870.

27. Alcala, J. R., E. Gratton, and F. G. Prendergast. 1987. Resolvability of fluorescence lifetime distributions using phase fluorometry. Biophys. J. 51:587-596.

28. Szczepanska-Konkel, M., A. N. K. Yusufi, and T. P. Dousa. 1987. Interactions of $\left[{ }^{14} \mathrm{C}\right]$-phosphonoformic acid with renal cortical brush-border membranes. Relationship to the $\mathrm{Na}^{+}$-phosphate cotransporter. J. Biol. Chem. 262:8000-8010.

29. Segel, I. H. 1976. Biochemical Calculations. 2nd ed. John Wiley and Sons, New York. 208-323.

30. Sokal, R. R., and J. F. Rohlf. 1969. Biometry: The Principles and Practice of Statistics in Biological Research. W. H. Freeman and Co., San Francisco.

31. DeSmedt, H., and R. Kinne: 1981. Temperature dependence of solute transport and enzyme activities in hog renal brush border membrane vesicles. Biochim. Biophys. Acta. 648:247-253.

32. Friedlander, G., M. Shahedi, C. LeGrimellec, and C. Amiel. 1988. Increase in membrane fluidity and opening of tight junctions have similar effects on sodium-coupled uptakes in renal epithelial cells. J. Biol. Chem. 263:11183-11188.

33. Lakowicz, J. R., F. G. Prendergast, and D. Hogen. 1979. Differential polarized phase fluorometric investigations of diphenylhexatriene in lipid bilayers. Quantitation of hindred depolarizing rotations. Biochemistry. 18:508-519.

34. Kutchai, H., L. H. Chandler, and G. B. Zavoico. 1983. Effects of cholesterol on acyl chain dynamics in multilamellar vesicles of various phosphatidylcholines. Biochim. Biophys. Acta. 736:137-149.

35. Sweet, W. D., W. G. Wood, and F. Schroeder. 1987. Charged anesthetics selectively alter plasma membrane order. Biochemistry. 26:2828-2835.

36. Karnovsky, M. J., A. M. Kleinfeld, R. L. Hoover, and R. D. Klausner. 1982. The concept of lipid domains in membranes. J. Cell Biol. 94:1-6.

37. Ives, H. E., and A. S. Verkman. 1985. Effects of membrane fluidizing agents on renal brush border proton permeability. Am. J. Physiol. 249(Renal Fluid Electrolyte Physiol. 18):F933-F940.

38. Saito, Y., and D. F. Silbert. 1979. Selective effects of membrane sterol depletion on surface function. Thymidine and 3-O-Methyl-Dglucose transport in a sterol auxotroph. J. Biol. Chem. 254:1 102-1 107.

39. Baldassare, J. J., Y. Saito, and D. F. Silbert. 1979. Effects of sterol depletion on LM cell sterol mutants. Changes in the lipid composition of the plasma membrane and their effects on 3-O-methylglucose transport. J. Biol. Chem. 254:1108-1113.

40. Wenzel, K., and H. Ebel. 1987. Effects of oleic acid on lipid order, mobility and $\mathrm{Na}^{+}$-D-glucose transport in rat renal brush border membranes. Xth ISN Congress. Satellite Symposium on Structure, Function, and Regulation of Membrane Transport Proteins. 98 (Abstr.).

41. Carriere, B., and C. LeGrimellec. 1986. Effects of benzyl alcohol on enzyme activities and D-glucose transport in kidney brushborder membranes. Biochim. Biophys. Acta. 857:131-138.

42. Molitoris, B. A., and R. Kinne. 1987. Ischemia induces surface membrane dysfunction. Mechanism of altered $\mathrm{Na}^{+}$-dependent glucose transport. J. Clin. Invest. 80:647-654.

43. Brasitus, T. A., R. Dahiya, P. K. Dudeja, and B. M. Bissonette. 1988. Cholesterol modulates alkaline phosphatase activity of rat intestinal microvillus membranes. J. Biol. Chem. 263:8592-8597.

44. Moss, D. W. 1984. Aspects of the relationship between liver, kidney, and bone alkaline phosphatases. In Human Alkaline Phosphatases. T. Stigbrand and W. H. Fishman, editors. Alan R. Liss, Inc., New York. 79-86. 